\title{
A System Dynamics Model Approach for Simulating Hyper-inflammation in Different COVID-19 Patient Scenarios
}

\author{
Vladimir Estivill-Castro ${ }^{3}{ }^{\mathrm{a}}{ }^{\mathrm{a}}$, Enrique Hernández-Jiménez ${ }^{1,2} \circledast^{\mathrm{b}} \mathrm{and}$ David F. Nettleton ${ }^{3} \mathbb{C l}^{\mathrm{c}}$ \\ ${ }^{1}$ Institut d'Investigació Biomèdica de Bellvitge, Barcelona, Spain \\ ${ }^{2}$ Loop Diagnostics S.L., Barcelona, Spain \\ ${ }^{3}$ Department of Information and Communications Technology (DTIC), Universitat Pompeu Fabra, Spain
}

\begin{abstract}
Keywords: Simulation of Biological Systems, Case Studies, Immune System Response, Model Construction, Rule-induction, Machine Learning.

Abstract: The exceptionally high virulence of COVID-19 and the patients' precondition seem to constitute primary factors in how pro-inflammatory cytokines production evolves during the course of an infection. We present a System Dynamics Model approach for simulating the patient reaction using two key control parameters (i) virulence, which can be "moderate" or "high" and (ii) patient precondition, which can be "healthy", "not so healthy" or "serious preconditions". In particular, we study the behaviour of Inflammatory (M1) Alveolar Macrophages, IL6 and Active Adaptive Immune system as indicators of the immune system response, together with the COVID viral load over time. The results show that it is possible to build an initial model of the system to explore the behaviour of the key attributes involved in the patient condition, virulence and response. The model suggests aspects that need further study so that it can then assist in choosing the correct immunomodulatory treatment, for instance the regime of application of an Interleukin 6 (IL-6) inhibitor (tocilizumab) that corresponds to the projected immune status of the patients. We introduce machine learning techniques to corroborate aspects of the model and propose that a dynamic model and machine learning techniques could provide a decision support tool to ICU physicians.
\end{abstract}

\section{INTRODUCTION}

Infectious pandemic corona-virus disease (COVID19), caused by severe acute respiratory disease Corona-virus 2 syndrome (SARS-CoV-2) is rapidly spreading worldwide (Salehi et al., 2020). In the case of COVID-19, a worsening has been observed from 7 to 8 days. However, this only occurs in some patients; and because of an over-reaction of the immune system (Pedersen and Ho, 2020). During this pandemic, the challenge is to diagnose those patients who are not getting worse; and thus free up space for those who need intensive care when they develop respiratory failure due to acute respiratory distress syndrome; the main cause of mortality. In a recent retrospective, multi-center study of 150 confirmed cases of COVID-19 in Wuhan, China, the authors suggest that mortality could be due to hyper-inflammatory sepsis (Chan et al., 2020).

\footnotetext{
a (iD) https://orcid.org/0000-0001-7775-0780

b (iD https://orcid.org/0000-0002-8232-8151

c (iD) https://orcid.org/0000-0002-5852-7716
}

After a coronavirus infection (COVID19, SARSCoV-2), patients with a more unfavourable course present a higher viremia; this viremia is also more persistent than those with a more benign course (Lescure et al., 2020). This fact will provoke a greater intensity in the inflammatory response and involvement of the different target organs. In clinical practice, we can monitor the evolution of the immune response by quantifying IL6, PCR, DD, troponin, LDH, and lymphopenia (Pedersen and Ho, 2020). From a conceptual point of view, the best therapeutic tool could be the early and aggressive use of specific antiviral treatments; but for those patients who evolved in an unfavourable way, the course of action seems to be an immunomodulatory treatment with increased healthcare resources (Yao et al., 2020). This care scheme is currently impossible, we do not have any effective antiviral treatment or tools that predict the evolution of patients in the early stages of the clinical picture, when patients are asymptomatic.

A first promising study (Gautret et al., 2020) showed that patients with COVID-19 treated with hy- 


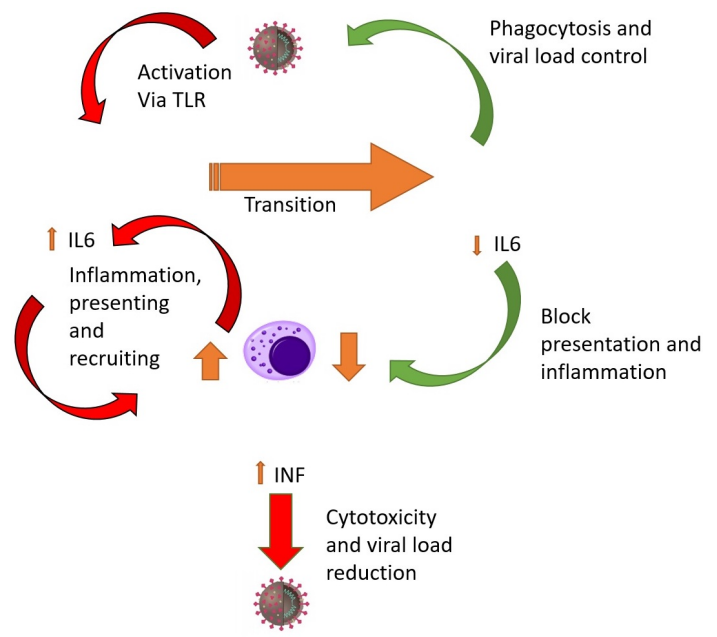

Figure 1: Schematic representation of the Immune System reaction to COVID.

droxychloroquine and azithromycin cured their infection and limited the transmission of the virus. However, a larger study showed limited potential and alerted of other possible side effects; thus their use is questionable (FDA cautions against use of hydroxychloroquine or chloroquine for COVID-19 outside of the hospital setting or a clinical trial due to risk of heart rhythm problems (FDA, 2020)). Additionally, Interleukin 6 (IL-6) inhibitor (tocilizumab) had been suggested for the treatment in COVID-19 (Zhang et al., 2020b; Choy et al., 2020). However, we still lack reliable studies and data to verify which patients will be beneficiaries of this treatment. Furthermore, the IL-6 inhibitor has potential hazards of inducing infectious diseases. Other work is also warranted to determine if these compounds could be useful as chemoprophylaxis to prevent virus transmission, especially for healthcare workers. Currently, some clinical trials have led researchers to claim that the COVID19 studies need new directions. However, there may also be an inadequate understanding of the key pathophysiological mechanisms operating in COVID19 patients. Several other clinical trials of immunomodulatory drugs have been initiated, thus corroborating the concept that immunosuppression has a central role.

The immune response to SARS-CoV-2 is the main cause of pulmonary pathology and associated morbidities that lead to death in a proportion of infected individuals, but this response is highly variable (Liu et al., 2020). There is limited knowledge of the kinetics, intensity, and diversity of cellular and humoral immune responses after SARS-CoV2 infection (Zhang et al., 2020a; Siracusano et al., 2020). The innate immune response in patients affected by a SARS-CoV-2 infection is characteristic of disease outcomes (Merad and Martin, 2020). We aim to model host immune signatures that can distinguish clinical outcomes in patients with COVID-19. How the immune system reacts in these early stages of coronavirus infection (COVID19, SARS-CoV-2) when patients have not developed severe symptoms has not been well studied (Siracusano et al., 2020).

In Section 2 we present a System Dynamics Model approach for simulating the patient reaction to viral infection. In particular, since the exceptionally high virulence of COVID-19 and the patients' precondition seem to constitute primary factors we focus on using two key control parameters (i) virulence, which can be "moderate" or "high" and (ii) patient precondition, which can be "healthy", "not so healthy" or "serious preconditions". We aim to reflect in the model the effects of pro-inflammatory cytokines produced and how macrophage polarisation evolves during the course of an infection. In particular, we focus on simulating the behaviour of Inflammatory (M1) Alveolar Macrophages, IL6 and Active Adaptive Immune system as indicators of the immune system response, together with the COVID viral load over time. Section 3 presents results of simulations and confirms that it is possible to build an initial model of the system to explore the behaviour of the key attributes involved in the patient condition, virulence and response. Section 4 concludes the paper with a discussion of some specific aspects of the model and the results, also in the context of related work.

\section{MODEL DEFINITION AND SIMULATION}

We introduce a System Dynamics Model for viral infection of the lungs calibrated with data from clinical studies (Yang et al., 2020; Hotchkiss et al., 2013). The modelling process, simulations, and optimisation analyses were performed using Vensim $囚 P L E$ software, version 8, academic license (Ventana Systems, Harvard, MA, USA). The model consists of interconnected "stocks" and "flows" to represent the immune system behaviour in response to a COVID infection. There has been a strong interest in epidemiological models for COVID-19 that study the propagation of the virus and the potential impact of measures, such as social distancing, to assist decision-making for managing transmission, delivery of testing and other resource management challenges (Currie et al., 2020). In particular, a freely distributed epidemiological model (vensim.com/coronavirus/) has attracted significant social-media attention. We take a rather orthogonal approach and apply dynamic system modelling to the reaction of the Immune System to viru- 
lent viruses such as COVID.

One of the fundamental contributions of defining this model is that it documents the interactions and potential cause-effect relationships between the many substances, proteins, and elements of tissue and of the immune system. A model of this type also highlights the potential gaps to increase research efforts as well as the points where a cause-effect relationship can be altered in search for vaccines or cures.

For instance, there is some understanding about how a coronavirus uses its $\mathrm{S}$ protein to enter into host cells (mainly latching to ACE-2). There is also some understanding of the replication processes that happen afterwards (which involves viral particles germinating into the endoplasmic reticulum-Golgi intermediate compartment). Some aspects are also known about how some new copies of the virus are released without immediate destruction of the host cell. Similarly, some microbiology understanding exists for how the infected cell presents antigen to antigen presentation cells. We remark that the details for COVID-19 are less known (Li et al., 2020) and those details now suggest differences between SARS$\mathrm{CoV}$ and MERS-CoV. Therefore, we will not simulate the details of antigen presentation here. We focus on the transitions and activity illustrated in Figure 1. Figure 2 contains the complete model with the main control input on the bottom left (virulence and patient precondition) and 9 main stockflows groups which have been designated as A to I. (see Table 1 for descriptions). A key stock generated in the model is "viral load" (C) and other key outputs are the immune system response $(\mathrm{F})$, lung tissue destruction (coming from $\mathrm{A}$ ) and macrophages (coming from E). The rectangles signify a "stock" (store of something), the circles represent a "rate", the hexagons are inputs, arrows are flows, variables (named source nodes) and the small clouds represent external source/destination. Table 2 provides a summary of the event sequence that the stock-flow model captures.

\subsection{Alveolar Macrophages (M1 and M2) and Viruses: Three Central Stocks}

The main player of the immune response in COVID19 are the alveolar macrophages (AM) (Merad and Martin, 2020). These cells detect and respond to danger signals resulting from pathogen colonization, growth, and tissue damage. Upon recognition, AM cells trigger an inflammatory response (M1). However, this reaction must be tightly regulated, as uncontrolled inflammation leads to clinical complications (Álvarez et al., 2017). One of the most impor- tant mechanisms to protect a host from excessive inflammation is a type of tolerance known as a refractory state or M2 (Rackov et al., 2016). This protective mechanism could also become a serious clinical problem, since an inadequate innate response significantly increases the risk of infections spreading. This crucial process, should never be confused with "immune paralysis". The M2 state or refractory state, is characterized by the ability of reduction of the inflammatory response (low levels of IL-6) and the increment of their phagocytosis capacity (Cubillos-Zapata et al., 2014). Although macrophage polarisation in humans is arguably a continuum (Atri et al., 2018), we create three stocks whose units are "macrophages".The interactions of these stocks and their flows is represented by Group E in Figure 2 and Table 1.

\subsection{Lung Tissue Fights Virus Infection}

We further our model by incorporating the signals between tissue cells. Interferon (IFN) is a generic name for signalling proteins produced and released by infected host cells in response to the presence of several viruses. The virus-infected cells produce interferon causing neighbour cells to intensify micro-biological anti-viral defences.

Thus, our model considers this neighbour defence system (refer to Group A in Figure 2 and Table 1). It represents that IFN released by infected lung tissue results in a reduction in the rate by which viruses transform healthy lung cells to infected cells. We do not intend to be too detailed in the impact of the virus with respect to different types of interferon. Suffice to say that there is some consensus that the IFN response is high in mild-to-moderate patients, but is reduced in the severe cases (Hadjadj et al., 2020).

To elaborate the model, we progress to represent the cytopathic effects and the viral evasion capability that appears in viral infections from human coronaviruses (SARS-Cov, MERS-CoV and COVID-19). It is clear that viral evasion contributes to the severity of the disease, but all the processes that lead to a dysregulated immune response and sustained inflammation are not completely understood (Channappanavar and Perlman, 2017). While there are several commonalities between the viruses' mechanisms for evasion in COVID-19, SARS-CoV and MERS-CoV (Li et al., 2020), there are also some differences and several details unknown (Pedersen and Ho, 2020). Therefore, we consider that our model incorporates all these aspects by the viruses impacting on the IFN-immune response modelled earlier. That is, we represent the interference the virus performs affecting interferon signals to neighbour tissue cells. Thus, higher virulence 


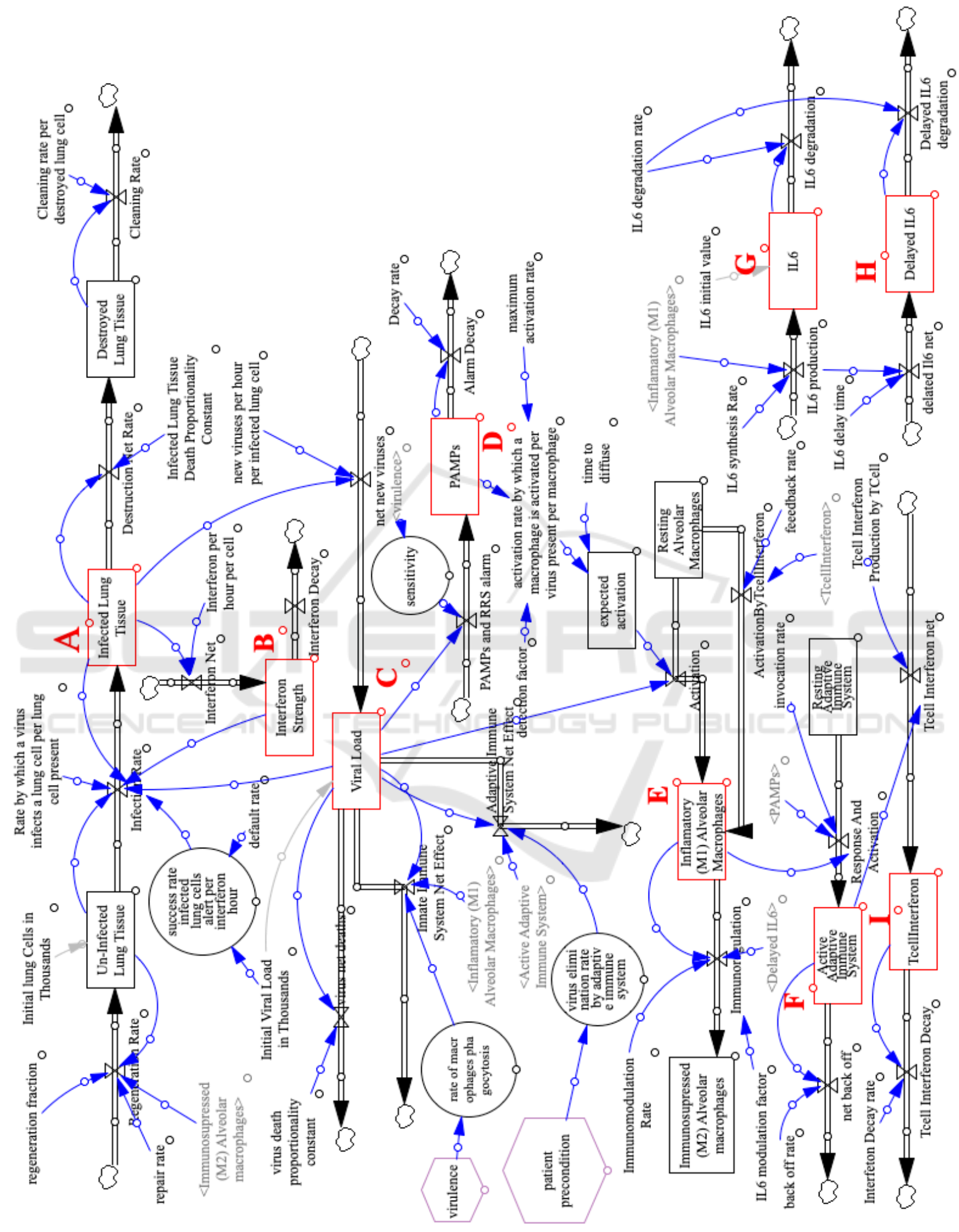

Figure 2: System Dynamics Model for the Immune system response to COVID. The rectangles signify a "stock" (quantity existing at a point in time), the circles represent a "rate", the magenta hexagons are inputs, arrows are flows and the small clouds represent external source/destination. 
Table 1: Stock-flow groups as defined in Fig 2.

\begin{tabular}{||l|l||}
\hline \hline \multicolumn{1}{|c|}{ Stock-flow groups \& Description } & \multicolumn{1}{|c||}{ Links in-out to other stock-flow group } \\
\hline \hline A $:$ Lung tissue (uninfected $\rightarrow$ infected $\rightarrow$ destroyed) & Outgoing link to Flow C, incoming links from \{Flow C,Flow E $\}$ \\
\hline B : Interferon strength & Outgoing link to Flow A, incoming link from Flow A. \\
\hline C : Viral load & Outgoing links to \{Flow A, Flow D, Flow E\}, incoming link from Flow A \\
\hline D : PAMPS & Outgoing link to Flow E, incoming link from Flow C \\
\hline E : Alveolar macrophages (resting $\rightarrow$ inflammatory(M1) $\rightarrow$ immunosuppressed(M2)) & Outgoing links to \{Flow F, Flow G\}, incoming links from $\{$ Flow C, Flow D $\}$ \\
\hline F : Adaptive immune system resting $\rightarrow$ active & Incoming link from Flow E, outgoing link to Flow I \\
\hline G : IL6 (production $\rightarrow$ degradation) & Outgoing links to Flow H, incoming link from Flow G \\
\hline H : Delayed IL6 & Incoming links from Flow G \\
\hline I : T-cell Interferon & Incoming links from Flow F \\
\hline \hline
\end{tabular}

means less effective interferon signals.

The focus of model is to represent the link between amplified inflammatory response and higher virus replication (Channappanavar and Perlman, 2017). Therefore, our model represents (refer to Group C and Group D in Figure 2 and Table 1) the belief that higher viral titters result in an increased viral Pathogen-Associated Molecular Patterns (PAMPs) that further dampen IFN signalling and stimulate Pattern Recognition Receptors (PRRs).

\subsection{Macrophage Activation}

Macrophages detect viruses in many ways. One of the nasty aspects of coronaviruses such as COVID19, SARS-CoV and MERS-CoV, is that they impact the role of Pattern Recognition Receptors (PRRs)in the innate immune system response (Li et al., 2020). For simplicity, we model that PRRs are proteins expressed by macrophages (although also expressed by dendritic cells, monocytes, neutrophils and epithelial cells). They identify two types of molecules.

1. Pathogen-Associated Molecular Patterns (PAMPs): foreign molecules coming from microbial pathogens.

2. Damage-Associated Molecular Patterns (DAMPs): molecules coming from damaged or dead cells in the host.

As we mentioned, although macrophages exhibit a range of phenotypes, they can be predominantly separated into two types (Stout and Suttles, 2004).

- M1 macrophages appear in the early stages of inflammation and are activated by four key mediators: interferon- $\gamma(\mathrm{IFN}-\gamma)$, tumour necrosis factor (TNF), and damage associated molecular patterns (DAMPs). From these mediator molecules, alveolar macrophages create a pro-inflammatory response that in return produces pro-inflammatory cytokines like Interleukin-6 (IL-6 ${ }^{1}$ ).

\footnotetext{
${ }^{1}$ Interleukin: cytokines made by one leukocyte and acting
}

- M2 macrophages produce an anti-inflammatory response via the release of Interleukin-4 or Interleukin-13. They participate in tissue healing.

These two types of macrophages can be converted into each other (Liu et al., 2014). Interferon (IFN)- $\gamma$ activates resting macrophages as M1 macrophages. When fighting severe viral infections, more macrophages are recruited by the secretion of further inflammatory mediators (Liu et al., 2014).

Our model represents the migration between these two types. The model represents the late awakening of macrophages because of the virus capacity to block the signals that allow its early detection. It has been reported that coronaviruses are capable of circumventing detection by developing double-membrane vesicles without PRRs and then replicate in these vesicles ( $\mathrm{Li}$ et al., 2020). Alveolar macrophages are the crucial defence against viruses. Although macrophages defend against viruses in many ways (secretion of oxygen metabolites, lysozyme, antimicrobial peptides and proteases), our model will focus on phagocytosis and intracellular killing.

Thus, our model enables to represent the virulence of the virus by regulating the sensitivity; i.e. the capacity of its rapid detection. With early detection, (high sensitivity, low virulence), M1 macrophages grow quickly in numbers and extinguish the virus rapidly. The alternative scenario is when the virus can pass under the radar for a while, resulting in a slow response of low numbers of macrophages.

\subsection{Macrophages Production of IL-6}

We calibrate our model so it matches the observation that COVID-19 patients suffer from excess inflammation at 9-10 days (Zhou et al., 2020). Cytokines and chemokines have long been understood to have an important role in immunity and immunopathology, but exuberant and deregulated immune responses have

on other leukocytes 
Table 2: Event sequence for the model of Figure 2.

\begin{tabular}{|c|c|}
\hline Steps in the process represented by the model & $\begin{array}{l}\text { Main stock-flow } \\
\text { groups (Table 1) } \\
\text { associated with } \\
\text { chronological steps } \\
\end{array}$ \\
\hline $\begin{array}{l}\text { Virus present. The virus displays many PAMPS which activate Macrophages M0 to M1. } \\
\text { Model notes: Virus infects a lung cell (lung tissue), interferon is released which alerts neighbouring lung cells. Very low } \\
\text { loads and non aggressive/simple viruses do not progress from this. COVID- } 19 \text { can pass this stage. }\end{array}$ & $\begin{array}{l}\text { Flow A, Flow C, } \\
\text { Flow D and } \\
\text { Flow E }\end{array}$ \\
\hline $\begin{array}{l}\text { M1 produces inflammatory cytokines like IL- } 6 \text {. } \\
\text { Model notes: Virus passes the interferon alarm of lung cells to their neighbours, and grows by infecting other neighbouring } \\
\text { cells. Viruses may kill or generate copies before killing. Most human cells when infected by viruses generate alarm signals. } \\
\text { COVID-19 can reduce the detectability of these signals released by infected cells. }\end{array}$ & $\begin{array}{l}\text { Flow C, Flow B, } \\
\text { Flow E and } \\
\text { Flow G }\end{array}$ \\
\hline $\begin{array}{l}\text { M1 starts presenting the virus PAMPS and other antigens to T-cells (CD4 and CD8, and } \\
\text { then T cells start producing Interferon). } \\
\text { Model notes: Macrophages of lung tissue [eventually] detect the virus (generically by PAMPs and PPRS), become active } \\
\text { [turn into predominantly M1] and fight it. } \\
\text { 1. For many viruses and small loads the macrophages can kill all and destroy the infection. } \\
\text { 2. They themselves raise alarm signals to activate other Macrophages to turn M1 and to produce inflammation (release IL-6 } \\
\text { and TNS which are pro-inflammatory cytokines ) } \\
\text { 3. This also creates exponential growth in the activation of macrophages of type M1, which if all goes well, can destroy } \\
\text { the virus, and once virus load is down, M1's turn into M2s, IL-6 comes down and the tissue regenerates because M2's } \\
\text { repair). Sustained inflammation and long time periods of IL-6 is destructive of other organs, sepsis, storm, etc } \\
\text { 4. Some of the macrophages are recruited by the release of IL-6 and its propagation in the body from other regions beyond } \\
\text { the infected tissue (the lung) } \\
\text { 5. Possibility that COVID-19 can have a stale-mate with a macrophage by infecting it into an abortive infection (the virus } \\
\text { also does not get to reproduce). }\end{array}$ & $\begin{array}{l}\text { Flow E, Flow D } \\
\text { and Flow B } \\
\text { Sub-steps } \\
\text { (see Model Notes): } \\
1 \text { involves Flow C, } \\
\text { Flow E, Flow A } \\
2 \text { involves Flow E, } \\
\text { Flow G, Flow A } \\
3 \text { involves Flow E, } \\
\text { Flow H } \\
4 \text { involves Flow E }\end{array}$ \\
\hline $\begin{array}{l}\text { Interferon produces M1 and activated CD8 T cells and NK cells to destroy the virus. } \\
\text { Model notes: Several types of lymphocytes are produced rapidly and of all kinds in young people (slowly and with biases for } \\
\text { some types of T-cells). For elderly people, people with diabetes, people with already issues with their immune system [i.e. } \\
\text { lupus] the reinforcements of the adaptive immune system come slow, and/or with biases for some types of T-cells. . }\end{array}$ & Flow E \\
\hline $\begin{array}{l}\text { M1 becomes M2. } \\
\text { Model notes: Modelled via the immunosuppression rate. Typically, this is regulated via intracellular and genetic regulation } \\
\text { inside M1 macrophage. }\end{array}$ & Flow E \\
\hline $\begin{array}{l}\text { M2 stops producing inflammation (IL-6) and stops presenting } \\
\text { Model notes: This also implies that M2 stops presenting and blocks the T-cell response - because of this cause damage to the } \\
\text { lung. It also increases their phagocytic capacity to eliminate the virus. }\end{array}$ & Flow E, Flow H \\
\hline
\end{tabular}

been shown to potentially cause lung damage and decreased survival (Pedersen and Ho, 2020). We also model the observation that, in SARS-CoV-2 infected individuals, cytokines like IL-6 increase during disease and decreases during recovery. These cytokines are especially higher in patients requiring ICU admission which also exhibit significantly higher levels of fever (Yang et al., 2020). This inflammatory cytokine levels were slightly elevated or within the normal range in moderate cases, but remarkably elevated in most severe cases. These cytokines are likely produced by highly inflammatory macrophages that have been implicated in the cytokine storm. The discovery that several potent cytokines, including IL- 6 are in increased concentrations in COVID-19 patients, led to the concept of blocking them as a treatment to reduce a cytokine storm (Yao et al., 2020).

However, the behaviour of IL-6 in our model is a consequence of the macrophages production. Therefore, the next elaboration of our model is the response by the innate immune system (refer to Group $G$ and Group $\mathrm{H}$ in Figure 2 and Table 1). In light of the larger numbers of viruses, M1 macrophages produce cytokines (including Interleukin-1, IL-6, and tumour necrosis factor- $\alpha$ ), chemokines (including Interleukin-8), and arachidonic metabolites. How- ever, among many roles, we focus on IL-6's effect to induce inflammation (Tanaka et al., 2014). Moreover, IL-6's elevated values are consistent in COVID-19 patients, especially those with severe outcomes (Zhou et al., 2020; Chen et al., 2020b). IL-6 is believed to be central to COVID-19 infections resulting in dysfunctional immune response sometimes considered similar to sepsis (Hotchkiss et al., 2013) or a so-called cytokine storm (Chen et al., 2020a). In our model, we focus on IL-6 production. Again, we are aware that many other phenomena happen concurrently, in order for the binding of PAMPs to Toll-Like Receptors (TLRs, which are a type of pattern-recognition receptors) to eventually result in the activation and detachment of alveolar macrophages from the alveolar epithelial cells, followed with the phagocytosis of viruses and secretion of Inflammatory cytokines (IL-6). However, for now such detail is not modelled. What is modelled is a predator-prey approach where activated (M1) macrophages hunt viruses.

Our model aims to simulate autocrine feedback loops that amplify inflammation (Choy and RoseJohn, 2017). Again, there other innate immune cells (such as neutrophils and monocytes) that produce and respond to IL-6 besides macrophages. but we focus on macrophages since studies have found that for 
COVID-19, there is a high incidence of macrophage infiltration (Channappanavar and Perlman, 2017).

\subsection{The Adaptive Immune System}

In our modeling of the adaptive immune system, we consider background information relating the key stocks. For example, the observation that COVID19 causes macrophages to become M1 type and produce the pro-inflammatory cytokine IL6 (Pedersen and Ho, 2020). In its turn, Il-6 promotes lymphocyte necrosis (Chen et al., 2020b). The serum concentration of IL-6 is negative to blood CD4 and CD8 T cells (Chen et al., 2020b). COVID-19 infection can cause T-cell exhaustion, and more noticeable with patients requiring ICU care (Diao et al., 2020). In terms of the effect of patient preconditions on the adaptive immune system, smoking and advanced patient age are key factors (Zhou et al., 2020; Diao et al., 2020; Feller et al., 2018). Tcell and B-cell function presents age-dependent defects that can result in an over-production of type 2 cytokines, which in turn results in deficient control of viral infection and prolonged proinflammatory responses (Zhou et al., 2020). Smoking has been identified as a factor that promotes macrophage activation and the macrophage polarisation for M1 macrophages over M2 macrophages (Feller et al., 2018). Elderly patients exhibit lower T-cell numbers, with suggests that TNF- $\alpha$ could be inducing T-cell depletion (Diao et al., 2020). For our modelling purposes, we introduce the condition of the patient as an element that distorts the engagement of the adaptive immune system (refer to Group F in Figure 2 and Table 1).

\section{RESULTS}

Our model offers two key control parameters, virus virulence and patient preconditions. The virus virulence (denoted $v$ ) is set to 1 or 2 , where 1 represents a virus which is not so virulent, generating many PAMPs, and not so adept at stealth; 2 indicates a high virulence with low sensitivity.

The patient pre-condition (denoted $p$ ) can be 0,1 or 2: 0 indicates a very healthy patient whose adaptive immune system supports the innate immune system with quick response; 1 indicates a not so healthy patient where the adaptive immune system is somewhat slower; 2 indicates a patient with very serious preconditions compromising the response of the adaptive immune system.

The six possible combinations of virus virulence and patient pre-conditions described in the design of
Table 3: Six experiments conducted with our model.

\begin{tabular}{||l||l|l|l|l|l|l||}
\hline \hline Virus virulence & 1 & 1 & 1 & 2 & 2 & 2 \\
\hline Patient preconditions & 0 & 1 & 2 & 0 & 1 & 2 \\
\hline \hline
\end{tabular}

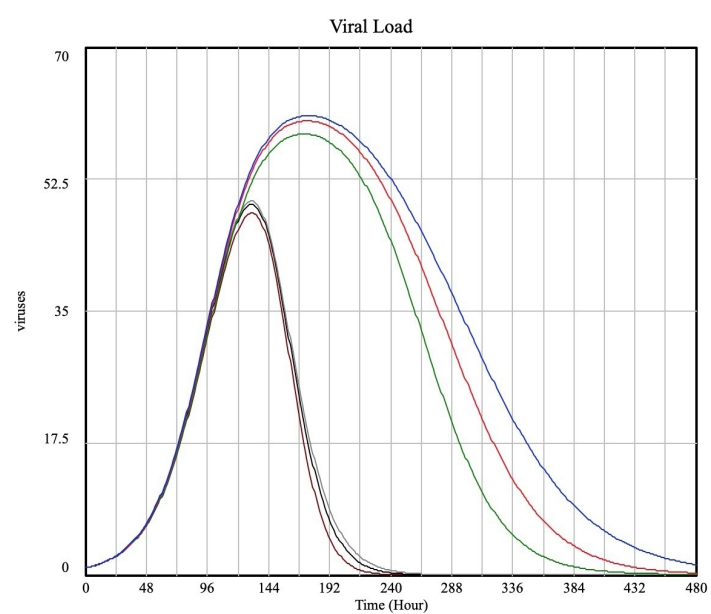

Virulence 2 Patient precondition $2-$ Virulence 1 Patient precondition 2 Virulence 2 Patient precondition 1 Virulence 1 Patient precondition 1 Virulence 1 Patient precondition 0

Figure 3: Plot of 'Viral Load' for different virulence and patient preconditions.

experiment are given in Table 3.

\subsection{Results of Simulation}

Figure 3 to Figure 5 show the plots for selected key stocks and rates for the model shown in Figure 2 and the experiments listed in Table 3. It can be seen that a healthy patient (patient precondition 0 ) is able to overcome a moderate and a high virus virulence. The macrophages, IL6 and adaptive immune system return to normal once the virus is overcome. However for the patient with serious precondition (patient precondition 2) the macrophages, IL6 and adaptive immune system stay high even when the virus virulence goes down. Also note that the less healthy the patient, the higher the values for macrophage, IL6 and immune system.

With reference to Figure 5 (a) (AAIS vs Viral Load) the log scale on the y-axis reveals an "attractor type" curve which can be an emergent feature of dynamical systems (Dudkowski et al., 2016). It can be seen that the viral load curves backwards for higher levels of AAIS (Active Adaptive Immune System). Note that this tendency is effectively following the sequence over time. In the case of Viral Load this makes sense - as the AAIS becomes more progressively activated, there is an inflection point around $x$-axis value 48 for the $v=1$ group and around the value 60 for the $v=2$ group. 
IL6

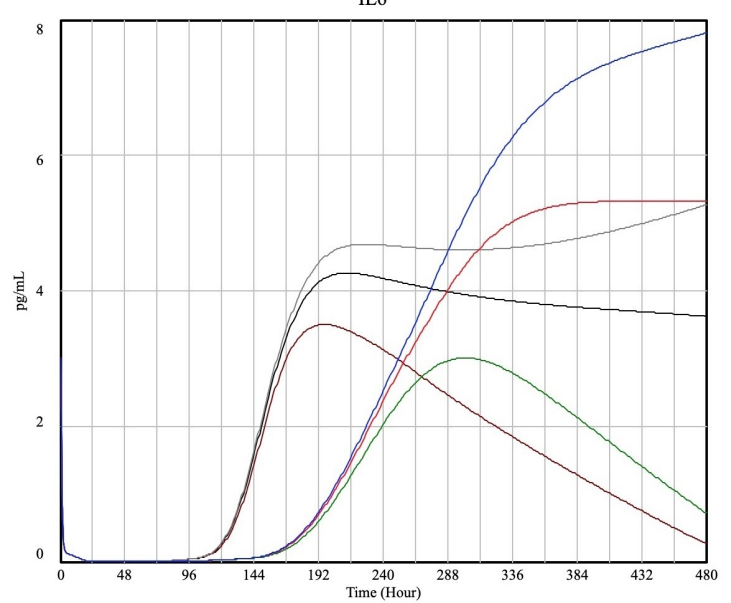

(a)

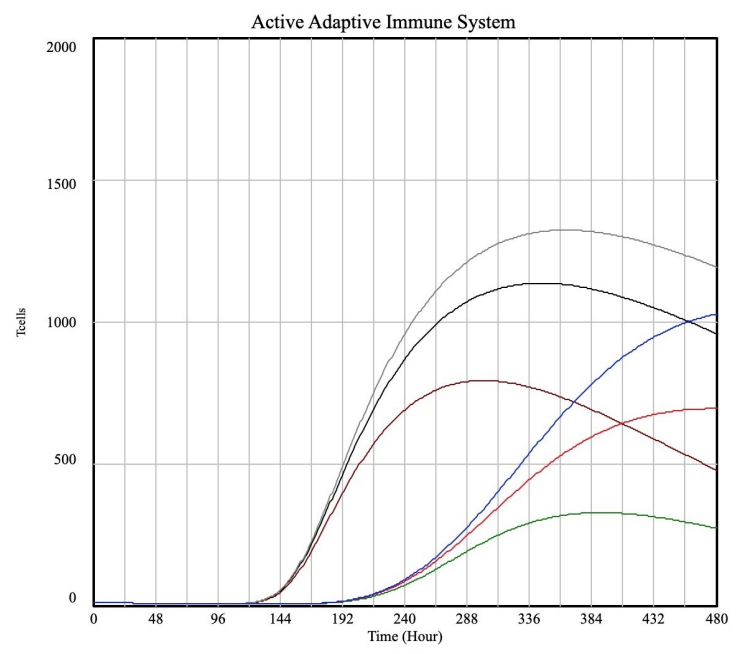

(b)

Virulence 2 Patient precondition 2
Virulence 2 Patient precondition 1 Virulence 1 Patient precondition 1 a

Figure 4: Plot of 'IL6' (a) and 'Active Adaptive Immune System' (b) vs time for different virulence and patient preconditions.

In the case of Figure 5 (b) (AAIS vs M1), something similar happens for the healthiest patients $(p=$ $0)$ as the immune system activates. For the highest virulence $(v=2)$ and worst patient condition $(p=2)$, M1 continues rising together with AAIS. Now consider the top right quadrant M1 $>45$ and AAIS $>700$ as a "dangerous region" (the thresholds represent an initial approximation which would be calibrated as future work). The intermediate virulence values and patient states are located to the left of this region with clear point $(v=1, p=1)$ for a positive infection outcome (going out of the "dangerous region"). But $v=1, p=2$ (worst patient type) is heading into the dangerous region, whereas $v=2, p=1$ is relatively static but also close to the dangerous region together with the two highest risk patient types.

\subsection{Interpretation, Confirmation and Limitations}

We interpret the displayed phenotype to have the following vivo relevance: a poor inflammatory capacity situation (High Virulence (id = 2) and Healthy Patient precondition $(\mathrm{id}=0)$ represented by the green lines in Figures 3 to 5) has impaired antigen presentation and would possibly either inhibit or alter the development of an adaptive response (AAIS). Moreover, an intermediate controlled level of M1 macrophages (black, grey and red lines, Figure 5(b)) reduce their inflammatory cytokines like IL-6. Such condition would contribute to protection against septic shock, and increased phagocytosis that would allow efficient viral clearance. In support of this, M2 macrophages have been related to decreased antigen presentation and impaired T-cell proliferation as well as reduced production of IFN. These observations underline the importance of the interaction between the antigenpresenting cells like M1 macrophages and the T-cells during infections. Finally, the higher inflammatory situation (blue lines in Figures 3 to 5) increases M1 and T-cell with more lag, and a lesser increasing slope; therefore they are much less efficient in eliminating the viral load.

Table 4 shows the correlations between all the stocks from the model of Figure 2. Correlations over 0.7 are highlighted, and high values can be seen between M1, IL6 and the Active adaptive immune system. Also, high values can be seen between M2 and Infected/destroyed lung tissue and Interferon strength. Apart from Time, Resting Alveolar has the most correlations greater than 0.7 with other stocks. The complete dataset was used to calculate the correlations and generate a PART rule-model appearing in Table 6. This rule model is set to predict IL6, was trained on a random sample of $50 \%$ of the data and tested on the remaining 50\%. PART is a highly effective rule-induction algorithm (Frank and Witten, 1998) and the Weka (Hall et al., 2009) implementation of a variation on Quinlan's C4.5 (Quinlan, 1993). This approach to build decision trees (or decision lists (Frank and Witten, 1998)) which uses Shannon's information theory to calculate an information gain to rank the best attribute candidates to include in the rules. The composition of the antecedents and consequents of the rules for predicting IL6 can give us useful information about what are the most rele- 


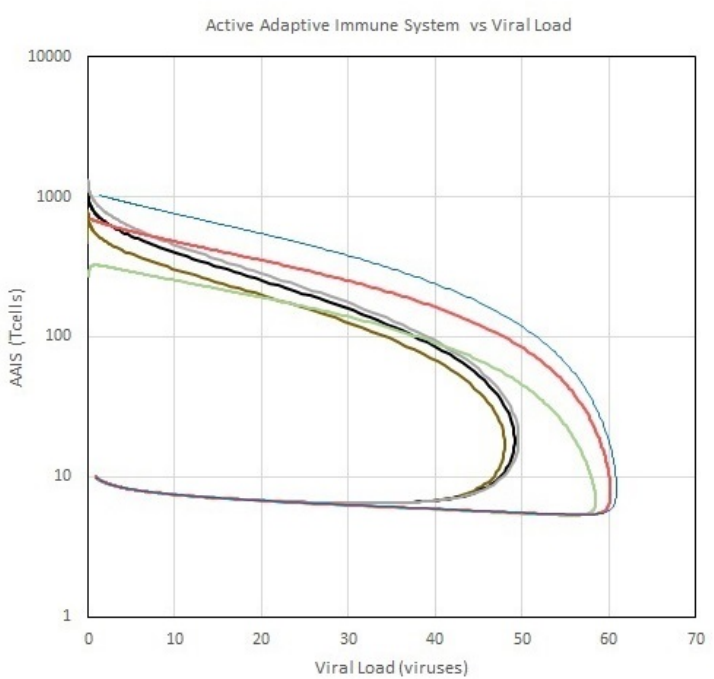

(a)

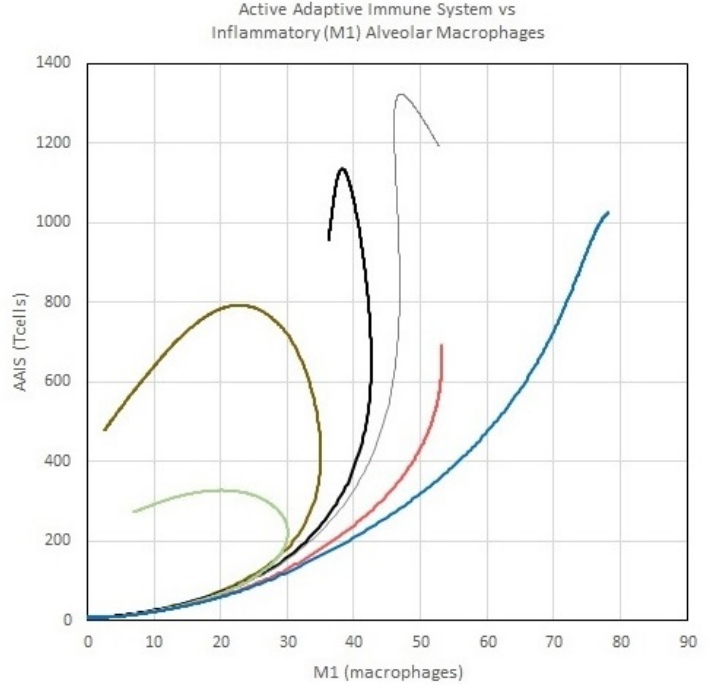

(b)

Virulence 2 Patient precondition 2
Virulence 2 Patient precondition 1 Virulence 2 Patient precondition 2

Virulence 1 Patient precondition 2
Virulence 1 Patient precondition 1

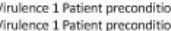

Figure 5: Plot of 'Active Adaptive Immune System' vs 'Viral Load' (a) and 'Inflammatory M1 Alveolar Macrophages" (b) for different virulence and patient preconditions.

Table 4: Correlations between model stocks.

\begin{tabular}{|c|c|c|c|c|c|c|c|c|c|c|c|c|c|c|c|c|c|}
\hline & 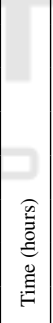 & 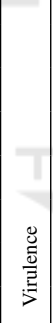 & 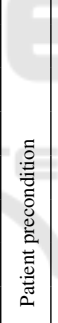 & 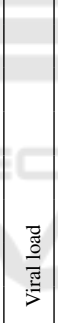 & 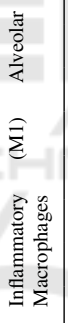 & 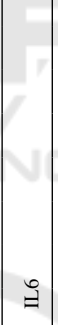 & 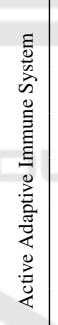 & 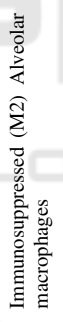 & 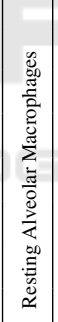 & 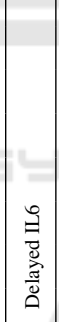 & 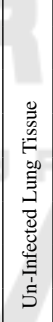 & 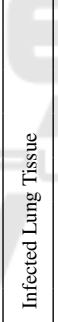 & 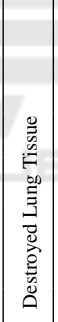 & 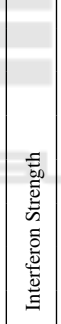 & $\sum_{a}^{\infty}$ & 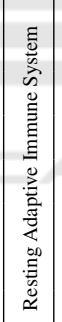 & 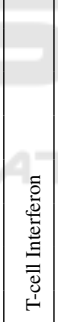 \\
\hline Time (hours) & 1 & 0 & 0 & -0.37 & 0.71 & 0.71 & 0.74 & 0.95 & -0.92 & 0.72 & -0.65 & -0.62 & -0.6 & 0.8 & 0.25 & -0.77 & 0.76 \\
\hline virulence & 0 & 1 & 0 & 0.39 & -0.03 & -0.03 & -0.41 & -0.05 & 0.04 & -0.03 & -0.13 & 0.04 & 0.04 & 0.08 & -0.46 & 0.42 & -0.41 \\
\hline Patient precondition & 0 & 0 & 1 & 0.07 & 0.36 & 0.36 & 0.23 & -0.14 & -0.13 & 0.34 & -0.03 & 0.01 & 0.01 & 0.01 & 0.06 & -0.19 & 0.16 \\
\hline Viral load & -0.37 & 0.39 & 0.07 & 1 & -0.37 & -0.38 & -0.62 & -0.41 & 0.43 & -0.42 & -0.26 & 0.41 & 0.47 & 0.1 . & -0.04 & 0.6 . & -0.58 \\
\hline $\begin{array}{l}\text { Inflammatory (M1) Alveo- } \\
\text { lar Macrophages }\end{array}$ & 0.71 & $-0.03 \mid$ & 0.36 & -0.37 & 1 & 1 & 0.75 & 0.62 & $|-0.9|$ & 1 & -0.6 & -0.6 & -0.58 & $|0.66|$ & 0.5 & -0.64 & 0.57 \\
\hline IL6 & 0.71 & -0.03 & 0.36 & -0.38 & 1 & 1 & 0.76 & 0.62 & $-0.9 \mid$ & & -0.59 & -0.6 & -0.59 & 0.65 & 0.49 & -0.65 & 0.58 \\
\hline $\begin{array}{l}\text { Active Adaptive Immune } \\
\text { System }\end{array}$ & 0.74 & -0.41 & 0.23 & -0.62 & 0.75 & 0.76 & 1 & 0.65 & -0.78 & 0.78 & -0.38 & -0.56 & -0.56 & 0.52 & 0.4 & -0.96 & 0.93 \\
\hline $\begin{array}{l}\text { Immunosuppressed (M2) } \\
\text { Alveolar macrophages }\end{array}$ & 0.95 & -0.05 & -0.14 & -0.41 & 0.62 & 0.62 & 0.65 & 1 & -0.9 & 0.63 & -0.64 & -0.69 & -0.68 & 0.78 & 0.34 & -0.67 & 0.65 \\
\hline $\begin{array}{ll}\text { Resting } & \text { Alveolar } \\
\text { Macrophages } & \\
\end{array}$ & -0.92 & 0.04 & -0.13 & 0.43 & -0.9 & -0.9 & -0.78 & -0.9 & 1 & -0.91 & 0.69 & 0.72 & 0.7 & -0.8 & -0.47 & 0.73 & -0.68 \\
\hline Delayed IL6 & 0.72 & -0.03 & 0.34 & -0.42 & 1 & 1 & 0.78 & 0.63 & -0.91 & 1 & -0.58 & -0.6 & -0.59 & 0.65 & 0.47 & -0.67 & 0.6 \\
\hline Un-Infected Lung Tissue & -0.65 & -0.13 & -0.03 & -0.26 & -0.6 & -0.59 & -0.38 & -0.64 & 0.69 & -0.58 & 1 & 0.44 & 0.37 & -0.97 & -0.64 & 0.3 . & -0.25 \\
\hline Infected Lung Tissue & -0.62 & 0.04 & 0.01 & 0.41 & -0.6 & $\mid-0.6$ & -0.56 & -0.69 & 0.72 & -0.6 & 0.44 & 1 & 0.99 & -0.52 & -0.35 & 0.52 . & -0.48 \\
\hline Destroyed Lung Tissue & -0.6 & 0.04 & 0.01 & 0.47 & -0.58 & -0.59 & -0.56 & -0.68 & 0.7 . & -0.59 & 0.37 & 0.99 & 1 . & -0.46 & -0.3 & 0.52 . & -0.48 \\
\hline Interferon Strength & 0.8 & 0.08 & 0.01 & 0.1 & 0.66 & 0.65 & 0.52 & 0.78 & -0.8 & 0.65 & -0.97 & -0.52 & -0.46 & 1 & 0.58 & -0.47 & 0.43 \\
\hline PAMPs & 0.25 & -0.46 & 0.06 & -0.04 & 0.5 & 0.49 & 0.4 & 0.34 & -0.47 & 0.47 & -0.64 & -0.35 & -0.3 & 0.58 & & -0.25 & 0.17 \\
\hline $\begin{array}{l}\text { Resting Adaptive Immune } \\
\text { System }\end{array}$ & -0.77 & 0.42 & -0.19 & 0.6 & -0.64 & -0.65 & -0.96 & -0.67 & 0.73 & -0.67 & 0.3 & 0.52 & 0.52 & -0.47 & $-0.25 \mid$ & 1 . & $|-0.99|$ \\
\hline T-cell Interferon & 0.76 & -0.41 & 0.16 & -0.58 & 0.57 & 0.58 & 0.93 & 0.65 & -0.68 & 0.6 & -0.25 & -0.48 & -0.48 & 0.43 & 0.17 & 0.99 & \\
\hline
\end{tabular}


Table 5: Two sample rules for predicting IL6.

\begin{tabular}{|c|c|c|}
\hline Rule 1 & $\begin{array}{l}\text { IF } \\
\text { THEN }\end{array}$ & $\begin{array}{l}\text { Inflammatory (M1) Alveolar Macrophages }>16.289 \\
\text { IL6 }= \\
0.1 \times \text { Inflammatory (M1) Alveolar Macrophages } \\
+0.0021 \times \text { Immunosupressed (M2) Alveolar macrophages } \\
-0.1056[763 / 0.931 \%]\end{array}$ \\
\hline $\mathrm{R}$ & $\begin{array}{l}\text { IF } \\
\text { THEN }\end{array}$ & $\begin{array}{l}\text { Inflammatory (M1) Alveolar Macrophages } \leq 1.381 \\
\text { IL6 }= \\
-0.0001 \times \text { Viral Load } \\
+0.1025 \times \text { Inflammatory (M1) Alveolar Macrophages } \\
-0.0101 \times \text { Delayed IL6 } \\
-0.0001 \times \text { T-cell Interferon } \\
+0.0019[410 / 1.095 \%]\end{array}$ \\
\hline
\end{tabular}

vant attributes to include in the model as proposed by the rule-induction algorithm.

From the complete model definition it can be seen that in the antecedent of the first three rules, "Inflammatory (M1) Alveolar Macrophages" is included. Rule 4 also uses Active Adaptive Immune System as well as M1. Rule 5 uses T-cell Interferon in the antecedent. In the antecedents, Rule 6 has "Time (Hour)" and "Active Adaptive Immune System", Rule 7 has "Viral Load" and Rule 8 has "Time (Hour)". On the other hand, the consequents of all the rules use mainly the following stocks: "Viral Load", "Time (Hour)", "M1", "Active Adaptive Immune System" and "Delayed IL6". It can be seen that Rule 1 and Rule 2 have the most corresponding cases, with 763 and 410, respectively. Rule 6 to Rule 9 have relatively much fewer cases associated. From the overall rule model (Appendix-Table 6), it can be concluded that "M1" and Active "Adaptive Immune System" are key stocks for predicting IL6.

The scope of the simulation is limited, for example, by fixing one of the key control parameters (i.e. viral load) while varying the other (patient preconditions). In this way we see the behaviour of the immune system through Figure 3, Figure 4 and Figure 5. On the other hand, the viral load does not vary for different patient preconditions and in vivo, we would expect a weaker patient to allow the virus load to be greater.

\section{DISCUSSION}

The results show that it is possible to build an initial model of the system to explore the behaviour of the key attributes involved in the patient condition, virulence and response. From the perspective of dynamic models, our results show that, with the few elements we have incorporated (relative to the many other players known to be involved), the system has many bifurcations and the behaviour is far from stable. In particular, such bifurcations inspire aspects that need further study. From the perspective of control theory, significant more information is required to exercise control of the inflammatory response by selecting medication depending on a modelled individualised classification of the potential outcome for each patient. The immune control of the excessive inflammatory response is called refractory state (RS); this mechanism is characterized by a significant reduction in the inflammatory capacity (IL-6) of innate immune cells like macrophages to a subsequent Pathogen-Associated Molecular Pattern (PAMP) challenge (López-Collazo and del Fresno, 2013; Agrawal et al., 2015). However, this state is not restricted to bacterial sepsis but has been observed for a number of pathologies such as acute pulmonary syndrome, cystic fibrosis, and even cancer (López-Collazo et al., 2010). While refractory state (RS) has been thought of as a protective mechanism against septic shock and ischemia, its immune regulation was associated with non-controlled hyperinflammatory status in COVID-19. Similarly, in acute pulmonary syndromes and cystic fibrosis, RS relates to an increased susceptibility to nosocomial infections (Biswas and Lopez-Collazo, 2009). Several studies have also shown some common mechanistic paradigms in RS across different diseases (LópezCollazo and del Fresno, 2013). In addition to this, RS in cystic fibrosis shows impaired antigen presentation, however it also displays a potent phagocytic activity. In cancer, tumor-associated macrophages (TAMs) show an immunosuppressive phenotype similar to RS macrophages. TAMs show decreased production of inflammatory cytokines like IL-6 but upregulation of anti-inflammatory cytokines (Cubillos-Zapata et al., 2014). This was explained by a defective NF-kB activation, overexpression of p50 NF-kB homodimers, and a functional TRIF pathway (Biswas and LopezCollazo, 2009).

Taken together, the SARS-CoV-2-induced cytokine storm is associated with disease severity and outcome. Understanding immune dysregulation in patients with COVID-19 not only provides a better understanding of the pathogenesis of SARS-CoV2, but also identifies targets for immune therapeutics (Merad and Martin, 2020). While antiviral agents are currently being explored, the use of antiviral agents alone may not be sufficient to stop the cytokine storm, lung destruction, and respiratory distress in patients who presented late after infection. Targeted immunomodulation that reduces cytokine storm can improve lung inflammation and hopefully reduce mortality (Avendaño Ortiz et al., 2017). Other studies of 
the viral factors driving immune dysregulation may provide insights into the conformation of vaccine responses to protective immunity (Zhang et al., 2020a). Advances in immunology and our understanding of the pathophysiological basis of sepsis provides exciting new therapeutic opportunities (Álvarez et al., 2017; Hotchkiss et al., 2013; Rackov et al., 2016; Shalova et al., 2015). We postulate that immunotherapy will have a wide range of beneficial effects on COVID19, and could be an important advance in infectious disease. Recently, interleukin 6 receptor (IL-6R) monoclonal antibody (tocilizumab)-directed COVID-19 therapy has been used in a clinical trial in China (No.ChiCTR2000029765) and the US (ClinicalTrials.gov Identifier: NCT04322773) Such therapy has been incorporated into COVID-19 management guidelines generated in China based on the concept of "cytokine storm" in COVID-19 pneumonia. Here, we explore the role of interleukin 6 (IL-6) in COVID-19 patients that could help the physician to recommend tocilizumab increasing the efficacy of this monoclonal antibody-directed therapy. Our results highlight the paramount need for future studies examining subsets and functions of both innate and adaptive immune cells at different time points, such as early during the asymptomatic viremic stage, during respiratory distress, and after recovery. This may identify mechanisms that lead to immune dysregulation in patients who have different susceptibilities to the disease, such as children versus the elderly. We now have a better understanding of the IL- 6 change and cytokine storm in COVID-19 pneumonia, but more data are needed on treatment options that improve survival.

\section{REFERENCES}

(2020). FDA cautions against use of hydroxychloroquine or chloroquine for covid-19 outside of the hospital setting or a clinical trial due to risk of heart rhythm problems - does not affect FDA-approved uses for malaria, lupus, and rheumatoid arthritis. FDA Drug Safety Communication: Safety Announcement [0424-2020].

Agrawal, A., Toledano, V., Hernández-Jiménez, E., Cubillos-Zapata, C., Flandez, M., Álvarez, E., VarelaSerrano, A., Cantero, R., Valles, G., García-Rio, F., and López-Collazo, E. (2015). Galactomannan downregulates the inflammation responses in human macrophages via $\mathrm{nfkb} 2 / \mathrm{p} 100$. Mediators of Inflammation, 2015:942517.

Álvarez, E., Toledano, V., Morilla, F., Hernández-Jiménez, E., Cubillos-Zapata, C., Varela-Serrano, A., CasasMartín, J., Avendaño Ortiz, J., Aguirre, L. A., Arnalich, F. Maroun-Eid, E., Martín-Quirós, A., Quintana Díaz, M., and López-Collazo, E. (2017). A sys- tem dynamics model to predict the human monocyte response to endotoxins. Frontiers in Immunology, 8:915.

Atri, C., Guerfali, F. Z., and Laouini, D. (2018). Role of human macrophage polarization in inflammation during infectious diseases. Int. J. of molecular sciences, 19(6): 1801.

Avendaño Ortiz, J., Maroun-Eid, C., Martín-Quirós, A., Toledano, V., Cubillos-Zapata, C., Gómez-Campelo, P., Varela-Serrano, A., Casas-Martin, J., LlanosGonzález, E., Alvarez, E., García-Río, F., Aguirre, L. A., Hernández-Jiménez, E., and López-Collazo, E. (2017). PD-L1 Overexpression During Endotoxin Tolerance Impairs the Adaptive Immune Response in Septic Patients via HIF1 $\alpha$. J. of Infectious Diseases, 217(3):393-404.

Biswas, S. K. and Lopez-Collazo, E. (2009). Endotoxin tolerance: new mechanisms, molecules and clinical significance. Trends in Immunology, 30(10):475 - 487.

Chan, J. F.-W., Yuan, S., Kok, K.-H., To, K. K.-W., Chu, H., Yang, J., Xing, F., Liu, J., Yip, C. C.-Y., Poon, R. W.-S., Tsoi, H.-W., Lo, S. K.-F., Chan, K.-H., Poon, V. K.-M., Chan, W.-M., Ip, J. D., Cai, J.-P., Cheng, V. C.-C., Chen, H., Hui, C. K.-M., and Yuen, K.-Y. (2020). A familial cluster of pneumonia associated with the 2019 novel coronavirus indicating person-toperson transmission: a study of a family cluster. The Lancet, 395(10223):514-523.

Channappanavar, R. and Perlman, S. (2017). Pathogenic human coronavirus infections: causes and consequences of cytokine storm and immunopathology. Seminars in Immunopathology, 39(5):529-539.

Chen, G., Wu, D., Guo, W., Cao, Y., Huang, D., Wang, H., Wang, T., Zhang, X., Chen, H., Yu, H., Zhang, X., Zhang, M., Wu, S., Song, J., Chen, T., Han, M., Li, S., Luo, X., Zhao, J., and Ning, Q. (2020a). Clinical and immunological features of severe and moderate coronavirus disease 2019. J. of Clinical Investigation, 130(5):2620-2629.

Chen, Y., Feng, Z., Diao, B., Wang, R., Wang, G., Wang, C., Tan, Y., Liu, L., Wang, C., Liu, Y., Liu, Y., Yuan, Z., Ren, L., and Wu, Y. (2020b). The novel severe acute respiratory syndrome coronavirus 2 (sars-cov-2) directly decimates human spleens and lymph nodes. medRxiv.

Choy, E. and Rose-John, S. (2017). Interleukin-6 as a multifunctional regulator: Inflammation, immune response, and fibrosis. J. of Scleroderma and Related Disorders, 2(2_suppl):S1-S5.

Choy, E. H., De Benedetti, F., Takeuchi, T., Hashizume, M., John, M. R., and Kishimoto, T. (2020). Translating il6 biology into effective treatments. Nature Reviews Rheumatology, 16(6):335-345.

Cubillos-Zapata, C., Hernández-Jiménez, E., Toledano, V., Esteban-Burgos, L., Fernández-Ruíz, I., Gómez-Piña, V., Del Fresno, C., Siliceo, M., Prieto-Chinchiña, P., Pérez de Diego, R., Boscá, L., Fresno, M., Arnalich, F., and López-Collazo, E. (2014). NFkB2/p100 is a key factor for endotoxin tolerance in human monocytes: a demonstration using primary human monocytes from patients with sepsis. J. of immunology, 193(8):4195-4202. 
Currie, C. S., Fowler, J. W., Kotiadis, K., Monks, T., Onggo, B. S., Robertson, D. A., and Tako, A. A. (2020). How simulation modelling can help reduce the impact of COVID-19. J. of Simulation, 14(2):83-97.

Diao, B., Wang, C., Tan, Y., Chen, X., Liu, Y., Ning, L., Chen, L., Li, M., Liu, Y., Wang, G., Yuan, Z., Feng, Z., Zhang, Y., Wu, Y., and Chen, Y. (2020). Reduction and functional exhaustion of $\mathrm{T}$ cells in patients with coronavirus disease 2019 (covid-19). Frontiers in Immunology, 11:827.

Dudkowski, D., Jafari, S., Kapitaniak, T., Kuznetsov, N., Leonov, G., and Prasad, A. (2016). Hidden attractors in dynamical systems. Physics Reports, 637:1-50.

Feller, D., Kun, J., Ruzsics, I., Rapp, J., Sarosi, V., Kvell, K., Helyes, Z., and Pongracz, J. E. (2018). Cigarette smoke-induced pulmonary inflammation becomes systemic by circulating extracellular vesicles containing wnt5a and inflammatory cytokines. Frontiers in immunology, 9:1724-1724.

Frank, E. and Witten, I. H. (1998). Generating accurate rule sets without global optimization. In Shavlik, J., editor, Fifteenth Int. Conference on Machine Learning, pages 144-151. Morgan Kaufmann.

Gautret, P., Lagier, J.-C., Parola, P., Hoang, V. T., Meddeb, L., Mailhe, M., Doudier, B., Courjon, J., Giordanengo, V., Vieira, V. E., Dupont, H. T., Honoré, S., Colson, P., Chabrière, E., Scola], B. L., Rolain, J.-M., Brouqui, P., and Raoult, D. (2020). Hydroxychloroquine and azithromycin as a treatment of covid-19: results of an open-label non-randomized clinical trial. Int. J. of Antimicrobial Agents, page 105949.

Hadjadj, J., Yatim, N., Barnabei, L., Corneau, A., Boussier, J., Pere, H., Charbit, B., Bondet, V., ChenevierGobeaux, C., Breillat, P., Carlier, N., Gauzit, R., Morbieu, C., Pene, F., Marin, N., Roche, N., Szwebel, T.A., Smith, N., Merkling, S., Treluyer, J.-M., Veyer, D., Mouthon, L., Blanc, C., Tharaux, P.-L., Rozenberg, F., Fischer, A., Duffy, D., Rieux-Laucat, F., Kerneis, S., and Terrier, B. (2020). Impaired type I interferon activity and exacerbated inflammatory responses in severe covid-19 patients. medRxiv.

Hall, M., Frank, E., Holmes, G., Pfahringer, B., Reutemann, P., and Witten, I. H. (2009). The weka data mining software: An update. SIGKDD Explor. Newsl., 11(1):10-18

Hotchkiss, R. S., Monneret, G., and Payen, D. (2013). Immunosuppression in sepsis: a novel understanding of the disorder and a new therapeutic approach. The Lancet. Infectious diseases, 13(3):260-268.

Lescure, F.-X., Bouadma, L., Nguyen, D., Parisey, M., Wicky, P.-H., Behillil, S., Gaymard, A., BouscambertDuchamp, M., Donati, F., Le Hingrat, Q., Enouf, V., Houhou-Fidouh, N., Valette, M., Mailles, A., Lucet, J.-C., Mentre, F., Duval, X., Descamps, D., Malvy, D., Timsit, J.-F., Lina, B., van-der Werf, S., and Yazdanpanah, Y. (2020). Clinical and virological data of the first cases of covid-19 in europe: a case series. The Lancet Infectious Diseases, 20(6):697-706.

Li, X., Geng, M., Peng, Y., Meng, L., and Lu, S. (2020). Molecular immune pathogenesis and diagnosis of covid-19. J. of Pharmaceutical Analysis, 10(2):102 -108 .
Liu, R., Han, H., Liu, F., Lv, Z., Wu, K., Liu, Y., Feng, Y., and Zhu, C. (2020). Positive rate of rt-pcr detection of sars-cov-2 infection in 4880 cases from one hospital in wuhan, china, from jan to feb 2020. Clinica Chimica Acta, 505:172 - 175 .

Liu, Y.-C., Zou, X.-B., Chai, Y.-F., and Yao, Y.-M. (2014). Macrophage polarization in inflammatory diseases. Int. J. of biological sciences, 10(5):520-529.

López-Collazo, E. and del Fresno, C. (2013). Pathophysiology of endotoxin tolerance: mechanisms and clinical consequences. Critical care (London, England), 17(6):242-242.

López-Collazo, E., Gómez-Piña, V., and Arnalich, F. (2010). Understanding immune dysfunctions in sepsis patients. Critical care (London, England), 14(4):435435.

Merad, M. and Martin, J. C. (2020). Pathological inflammation in patients with covid-19: a key role for monocytes and macrophages. Nature Reviews Immunology, 20(6):355-362.

Pedersen, S. F. and Ho, Y.-C. (2020). SARS-CoV-2: A storm is raging. J. of Clinical Investigation, 130(5):2202-2205.

Quinlan, J. (1993). C4.5: Programs for Machine Learning. Morgan Kaufmann Publishers, San Mateo, CA.

Rackov, G., Hernández-Jiménez, E., Shokri, R., CarmonaRodríguez, L., Mañes, S., Álvarez-Mon, M., LópezCollazo, E., Martínez-A, C., and Balomenos, D. (2016). P21 mediates macrophage reprogramming through regulation of p50-p50 NF-KB and IFN- $\beta$. $J$. of clinical investigation, 126(8):3089-3103.

Salehi, S., Abedi, A., Balakrishnan, S., and Gholamrezanezhad, A. (2020). Coronavirus disease 2019 (covid-19): A systematic review of imaging findings in 919 patients. American J. of Roentgenology, pages $1-7$.

Shalova, I. N., Lim, J. Y., Chittezhath, M., Zinkernagel, A. S., Beasley, F., Hernández-Jiménez, E., Toledano, V., Cubillos-Zapata, C., Rapisarda, A., Chen, J., Duan, K., Yang, H., Poidinger, M., Melillo, G., Nizet, V., Arnalich, F., López-Collazo, E., and Biswas, S. K. (2015). Human monocytes undergo functional re-programming during sepsis mediated by hypoxiainducible factor-1\&\#x3b1;. Immunity, 42(3):484498.

Siracusano, G., Pastori, C., and Lopalco, L. (2020). Humoral immune responses in covid-19 patients: A window on the state of the art. Frontiers in Immunology, 11:1049.

Stout, R. D. and Suttles, J. (2004). Functional plasticity of macrophages: reversible adaptation to changing microenvironments. J. of leukocyte biology, 76(3):509513.

Tanaka, T., Narazaki, M., and Kishimoto, T. (2014). Il-6 in inflammation, immunity, and disease. Cold Spring Harbor perspectives in biology, 6(10):a016295a016295.

Yang, X., Yu, Y., Xu, J., Shu, H., Xia, J., Liu, H., Wu, Y., Zhang, L., Yu, Z., Fang, M., Yu, T., Wang, Y., Pan, S., Zou, X., Yuan, S., and Shang, Y. (2020). Clinical course and outcomes of critically ill patients with sarscov-2 pneumonia in Wuhan, China: a single-centered, 
retrospective, observational study. The Lancet Respiratory Medicine, 8(5):475-481.

Yao, T.T. adn Qian, J. D., Zhu, W., Y., W., and Q., W. G. (2020). A systematic review of Lopinavir therapy for SARS coronavirus and MERS coronavirus - A possible reference for coronavirus disease-19 treatment option. J Med Virol., 92(6):556-563.

Zhang, J. J., Dong, X., Cao, Y. Y., Yuan, Y. D., Yang, Y. B., Yan, Y. Q., Akdis, C. A., and Gao, Y. D. (2020a). Clinical characteristics of 140 patients infected with SARS-CoV-2 in Wuhan, China. Allergy: European J. of Allergy and Clinical Immunology. Advance online publication.

Zhang, Y., Zhong, Y., Pan, L., and Dong, J. (2020b). Treat 2019 novel coronavirus (COVID-19) with il-6 inhibitor: Are we already that far? Drug Discoveries \& Therapeutics, 14(2):100-102.

Zhou, F. nd Yu, T., Du, R., Fan, G., Liu, Y., Liu, Z., Xiang, J., Wang, Y., Song, B., Gu, X., Guan, L., Wei, Y., Li, H., Wu, X., Xu, J., Tu, S., Zhang, Y., Chen, H., and Cao, B. (2020). Clinical course and risk factors for mortality of adult inpatients with COVID-19 in Wuhan, China: a retrospective cohort study. Lancet, 395:1054-1062.

\section{APPENDIX}

The datasets generated and analyzed for this study can be found in the Github available at: github.com/vladestivillcastro/SIMULTECH2021.

We also have available tables that provide details as follows.

Stocks: stock, equation and units.

Flows: flow, equation and units.

Auxiliary Variables: auxiliary variables, equation and units.

Constants ID, parameters, value, units.
Table 6: Learned rules.

\begin{tabular}{|c|c|}
\hline Rule 1 & $\begin{array}{l}\text { IF } \begin{array}{l}\text { Inflamatory (M1) Alveolar Macrophages }>16.289 \\
\text { THEN }\end{array} \text { IL6 }= \\
\quad 0.1 \times \text { Inflamatory (M1) Alveolar Macrophages } \\
\quad+0.0021 \times \text { Immunosupressed (M2) Alveolar macrophages } \\
\quad-0.1056[763 / 0.931 \%]\end{array}$ \\
\hline Rule 2 & \begin{tabular}{|ll} 
IF & Inflamatory (M1) Alveolar Macrophages $\leq 1.381$ \\
THEN & IL6 $=$ \\
& $-0.0001 \times$ Viral Load \\
& $+0.1025 \times$ Inflamatory (M1) Alveolar Macrophages \\
& $-0.0101 \times$ Delayed IL6 $-0.0001 \times$ T-cell Interferon \\
& $+0.0019[410 / 1.095 \%]$
\end{tabular} \\
\hline Rule 3 & 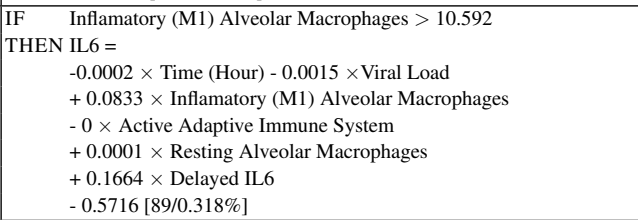 \\
\hline Rule 4 & \begin{tabular}{|l} 
IF \\
Active Adaptive Immune System $>9.922$ \\
Inflamatory (M1) Alveolar Macrophages $>6.424$ \\
THEN IL6 $=$ \\
$-0.0001 \times$ Time $($ Hour $)-0.0048 \times$ Viral Load \\
$+0.1134 \times$ Inflamatory $($ M1) Alveolar Macrophages \\
$-0.0003 \times$ Active Adaptive Immune System \\
$-0.0008 \times$ Immunosupressed (M2) Alveolar macrophages \\
$+0.0011 \times$ Resting Alveolar Macrophages \\
$-0.1284 \times$ Delayed IL6 $+0.0015 \times$ TcellInterferon \\
$-5.0631[69 / 0.096 \%]$
\end{tabular} \\
\hline Rule 5 & $\begin{array}{|ll|}\text { IF } & \text { TcellInterferon }>0.517 \\
\text { THEN } & \text { IL6 }= \\
& -0.0003 \times \text { Time }(\text { Hour })-0.0019 \times \text { Viral Load } \\
& +0.0992 \times \text { Inflamatory }(\mathrm{M} 1) \text { Alveolar Macrophages } \\
& +0.0001 \times \text { Active Adaptive Immune System } \\
& +0.1282[79 / 2.143 \%]\end{array}$ \\
\hline Rule 6 & $\begin{array}{ll}\text { IF } & \text { Time }(\text { Hour })>1.5 \text { and Active Adaptive Immune System } \leq 7.841 \\
\text { THEN IL6 }= \\
\quad-0.0003 \times \text { Time }(\text { Hour })-0.0103 \times \text { Viral Load }+0.7211[11 / 1.317 \%\end{array}$ \\
\hline Rule 7 & $\begin{array}{l}\text { IF } \quad \text { Viral Load } \leq 0.999 \\
\begin{array}{l}\text { THEN } \\
\quad+L 6= \\
\quad+0.9887[4 / 0 \%]\end{array}\end{array}$ \\
\hline Rule 8 & 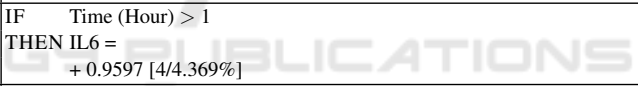 \\
\hline Rule 9 & $\begin{array}{r}\text { IF } \\
\text { THEN IL6 }= \\
+3[2]\end{array}$ \\
\hline
\end{tabular}

